\title{
Clinical Value of lncRNA LUCAT1 Expression in Liver Cancer and its Potential Pathways
}

\author{
Yan Jiao ${ }^{1}$, Yanqing $\mathrm{Li}^{2}$, Bai Ji ${ }^{1}$, Hongqiao Cai ${ }^{1}$, Yahui Liu ${ }^{1}$
}

1) Department of

Hepatobiliary and Pancreatic

Surgery, The First Hospital of Jilin University, Changchun, Jilin

2) Department of Pathophysiology, College of Basic Medical Sciences, Jilin University, Changchun, Jilin, P.R. China

Address for correspondence: Yahui Liu Department of Hepatobiliary and Pancreatic Surgery, The First Hospital of Jilin University, Changchun, Jilin Province, 130000, P.R. China liuyh_2008@yeah.net

\begin{abstract}
Background \& Aims: Emerging studies indicate that long noncoding RNAs (lncRNAs) play a role as prognostic markers in many cancers, including liver cancer. Here, we focused on the lncRNA lung cancer-associated transcript 1 (LUCAT1) for liver cancer prognosis.

Methods: RNA-seq and phenotype data were downloaded from the Cancer Genome Atlas (TCGA). Chisquare tests were used to evaluate the correlations between LUCAT1 expression and clinical features. Survival analysis and Cox regression analysis were used to compare different LUCAT1 expression groups (optimal cutoff value determined by ROC). The log-rank test was used to calculate the p-value of the Kaplan-Meier curves. A ROC curve was used to evaluate the diagnostic value. Gene Set Enrichment Analysis (GSEA) was performed, and competing endogenous RNA (ceRNA) networks were constructed to explore the potential mechanism. Results: Data mining of the TCGA -Liver Hepatocellular Carcinoma (LIHC) RNA-seq data of 371 patients showed the overexpression of LUCAT1 in cancerous tissue. High LUCAT1 expression was associated with age $(\mathrm{p}=0.007)$, histologic grade $(\mathrm{p}=0.009)$, T classification $(\mathrm{p}=0.022)$, and survival status $(\mathrm{p}=0.002)$. High LUCAT1 patients had a poorer overall survival and relapse-free survival than low LUCAT1 patients. Multivariate analysis identified LUCAT1 as an independent risk factor for poor survival. The ROC curve indicated modest diagnostic performance. GSEA revealed the related signaling pathways, and the ceRNA network uncovered the underlying mechanism.

Conclusion: High LUCAT1 expression is an independent prognostic factor for liver cancer.
\end{abstract}

Key words: IncRNA; LUCAT1 - liver cancer - prognosis - The Cancer Genome Atlas.

Abbreviations: ceRNA: competing endogenous RNA; DEG: differentially expressed genes; DEM: differentially expressed microRNA; GEAS: Gene Set Enrichment Analysis; lncRNA: long noncoding RNA; LUCAT1: lncRNA lung cancer-associated transcript 1; OS: overall survival; RFS: relapse-free survival; TCGA-LIHC: The Cancer Genome Atlas Liver Hepatocellular Carcinoma.

\section{INTRODUCTION}

Liver cancer is one of the most frequent malignant cancers with a poor prognosis despite some advances in surgical treatment and neoadjuvant therapy. The lack of useful markers makes it difficult for clinicians to predict clinical outcomes for liver cancer patients. Hence, novel markers need to be explored to identify liver cancer patients with poor prognosis and facilitate precision medicine.

Long noncoding RNAs (lncRNAs), a type of noncoding
RNA with a length of more than 200 nucleotides, have recently attracted increasing attention in the field of cancer [1]. Lung cancer-associated transcript 1 (LUCAT1), a novel lncRNA, is involved in smoking-related lung cancer [2]. A previous study demonstrated that LUCAT1 can be induced by cigarette smoke and is related to unfavorable prognosis in non-small cell lung cancer [2].

Recent studies on LUCAT1 have focused on different cancer types, including renal cancer [3], bladder cancer [4], glioma [5], osteosarcoma [6], colorectal cancer, and head and neck cancer [7]. However, the role of LUCAT1 in the prognosis of liver cancer remains unclear.

In the present study, we aimed to conduct data mining of The Cancer Genome Atlas Liver Hepatocellular Carcinoma (TCGA-LIHC) cohort data, evaluate potential clinical correlations, and evaluate the independent prognostic value of LUCAT1 for overall survival and relapse-free survival 
in liver cancer. In addition, we preliminarily explored the potential mechanism by conducting GSEA and constructing a competing endogenous RNA (ceRNA) network.

\section{METHODS}

\section{TCGA analysis}

RNA-seq data and phenotype data, including 321 tumors and 50 matched normal samples, were downloaded from The Cancer Genome Atlas (TCGA) (https://cancergenome.nih. gov/).

\section{GSEA}

Gene Set Enrichment Analysis (GSEA) is a computational method that determines whether a priori defined set of genes shows statistically significant, concordant differences between two biological states $[8,9]$. It was performed by using GSEA software 3.0 from the Broad Institute.

The expression data were RNA-seq data from TCGA-LIHC. The gene set of "h.all.v6.2.symbols.gmt" was downloaded from the Molecular Signatures Database (http://software. broadinstitute.org/gsea/msigdb/index.jsp). The normalized enrichment score (NES) was obtained with 1,000 permutations.

\section{Conduction of a ceRNA network}

Differentially expressed microRNAs and encoding genes were analyzed using the limma package [10]. The predicted miRNA-mRNA interactions were obtained from starBase v2.0 (http://starbase.sysu.edu.cn/starbase2/index.php) by default options $[11,12]$. The competing endogenous RNAs (ceRNA) network was constructed by merging differentially expressed genes (DEGs), differentially expressed microRNA (DEMs) and miRNA-mRNA interactions.

\section{Statistical analysis}

All statistical analyses and visualizations were carried out using R (version 3.5.1) [13]. The ggplot2 package was used to draw all boxplots and survival curves [14]. The ROC package was used to determine the optimal cutoff value of LUCAT1 expression [15]. The clinical correlation was tested by the Chisquare test using $\mathrm{R}$. The survival package was used to perform survival analysis and Cox analysis $[16,17]$. The $p$ value $<0.05$ was considered statistically significant.

\section{RESULTS}

\section{Patient characteristics}

The records of 371 patients with liver cancer were retrospectively reviewed from the TCGA database. The clinical records of patients included values for age, sex, histologic grade, stage, TNM classification, residual tumor, vital status, relapse, and LUCAT1 expression. Detailed information is provided in Table I.

\section{LUCAT1 expression in liver cancer}

As shown in the boxplot, LUCAT1 was overexpressed in cancerous tissues compared with normal tissues (Fig. 1). Interestingly, LUCAT1 was significantly differentially expressed in the different groups of stage, histologic grade, sex, and age (Fig. 1).
Table I. Baseline characteristics of patients with liver cancer

\begin{tabular}{|c|c|c|}
\hline \multicolumn{2}{|c|}{ Characteristics } & \multirow{2}{*}{$\begin{array}{c}\text { Number of patients } \\
(\%)\end{array}$} \\
\hline Age & $<55$ & \\
\hline & $\geq 55$ & $253(68.38)$ \\
\hline \multirow[t]{2}{*}{ Gender } & Female & $121(32.61)$ \\
\hline & Male & $250(67.39)$ \\
\hline \multirow[t]{3}{*}{ Histological type } & Fibrolamellar Carcinoma & $3(0.81)$ \\
\hline & Hepatocellular Carcinoma & $361(97.3)$ \\
\hline & Hepatocholangiocarcinoma & $7(1.89)$ \\
\hline \multirow[t]{5}{*}{ Histologic grade } & G1 & $55(14.82)$ \\
\hline & G2 & $177(47.71)$ \\
\hline & G3 & $122(32.88)$ \\
\hline & G4 & $12(3.23)$ \\
\hline & NA & $5(1.35)$ \\
\hline \multirow[t]{5}{*}{ Stage } & I & $171(46.09)$ \\
\hline & II & $86(23.18)$ \\
\hline & III & $85(22.91)$ \\
\hline & IV & $5(1.35)$ \\
\hline & NA & $24(6.47)$ \\
\hline \multirow[t]{6}{*}{$\mathrm{T}$ classification } & $\mathrm{T} 1$ & $181(48.79)$ \\
\hline & $\mathrm{T} 2$ & $94(25.34)$ \\
\hline & T3 & $80(21.56)$ \\
\hline & $\mathrm{T} 4$ & $13(3.5)$ \\
\hline & $\mathrm{Tx}$ & $1(0.27)$ \\
\hline & NA & $2(0.54)$ \\
\hline \multirow[t]{4}{*}{$\mathrm{N}$ classification } & No & $252(67.92)$ \\
\hline & N1 & $4(1.08)$ \\
\hline & $\mathrm{Nx}$ & $114(30.73)$ \\
\hline & NA & $1(0.27)$ \\
\hline \multirow[t]{3}{*}{ M classification } & M0 & $266(71.7)$ \\
\hline & M1 & $4(1.08)$ \\
\hline & $\mathrm{Mx}$ & $101(27.22)$ \\
\hline \multirow[t]{3}{*}{ Radiation therapy } & No & $338(91.11)$ \\
\hline & Yes & $8(2.16)$ \\
\hline & NA & $25(6.74)$ \\
\hline \multirow[t]{5}{*}{ Residual tumor } & R0 & $324(87.33)$ \\
\hline & $\mathrm{R} 1$ & $17(4.58)$ \\
\hline & $\mathrm{R} 2$ & $1(0.27)$ \\
\hline & $\mathrm{Rx}$ & $22(5.93)$ \\
\hline & NA & $7(1.89)$ \\
\hline \multirow[t]{2}{*}{ Vital status } & Deceased & $130(35.04)$ \\
\hline & Living & $241(64.96)$ \\
\hline \multirow[t]{2}{*}{ Relapse } & No & $179(56.29)$ \\
\hline & Yes & $139(43.71)$ \\
\hline \multirow[t]{2}{*}{ LUCAT1 } & High & $58(15.63)$ \\
\hline & Low & $313(84.37)$ \\
\hline
\end{tabular}

LUCAT1: lncRNA lung cancer-associated transcript 1; NA: not available.

\section{The clinical correlation of LUCAT1}

To explore the clinical correlation of LUCAT1, we used the Chi-square test and concluded that LUCAT1 expression 

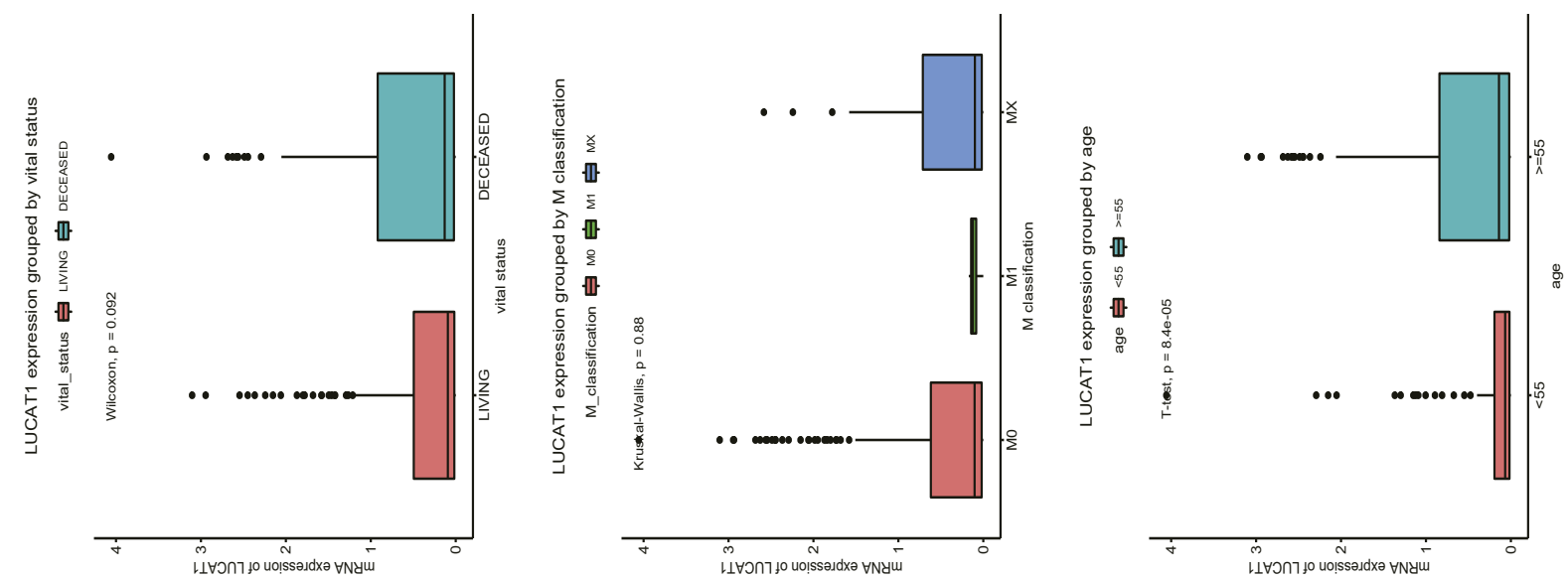

作
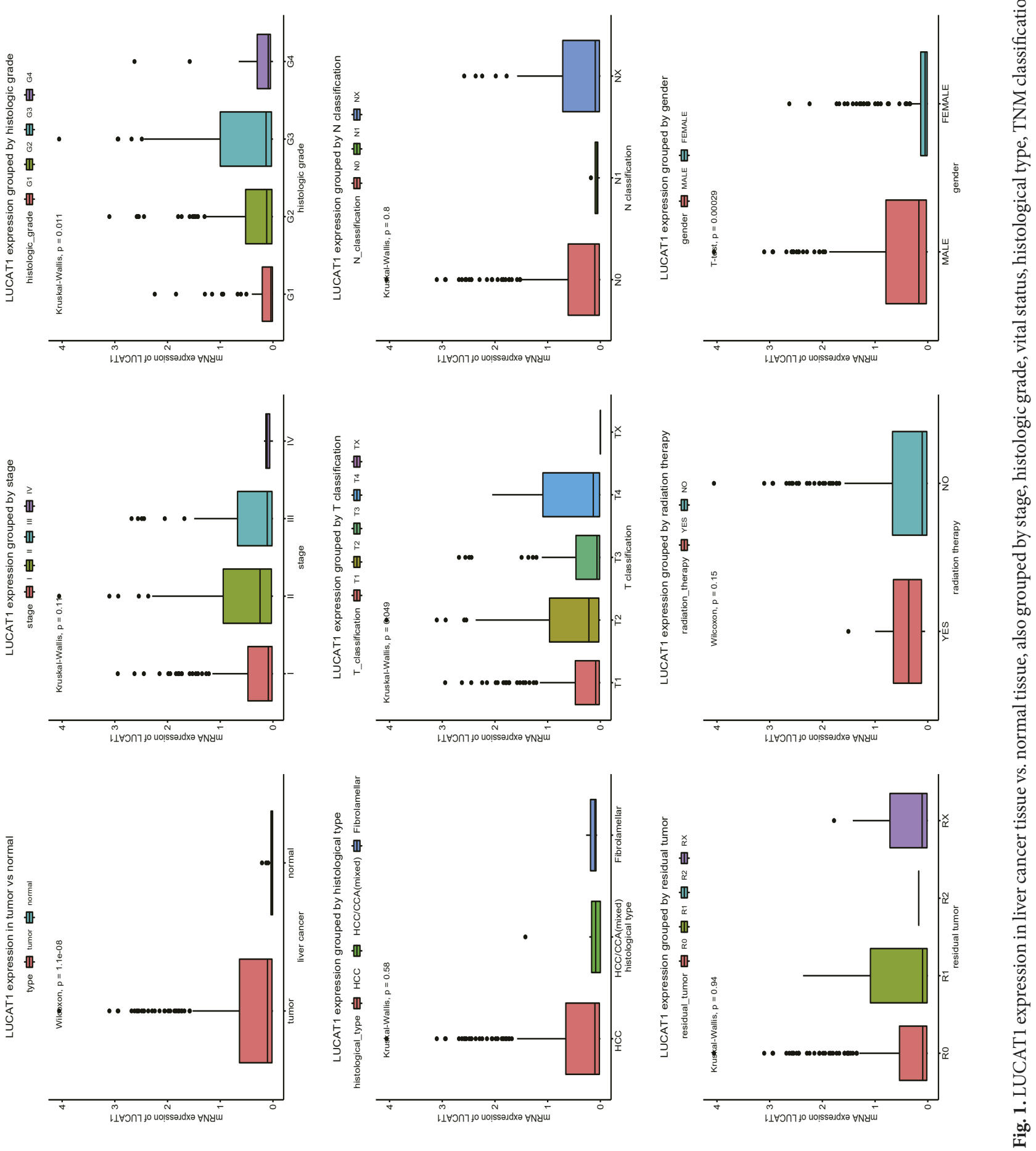
was correlated with age, histologic grade, T classification, and vital status (Table II).

\section{Impact of LUCAT1 expression on overall survival and} relapse-free survival in patients

To explore the impact of LUCAT1 expression on the prognosis of patients, we performed survival analysis and found that patients with high LUCAT1 expression had a poor overall survival and relapse-free survival (Figs. 2 and 3).

Subgroup analysis indicated that patients with high LUCAT1 expression in the stage G3/G4, stage I/II and male groups had shorter overall survival (Fig. 2). In addition, high
LUCAT1 expression showed a poor relapse-free survival in all groups except in the female group (Fig. 3).

\section{High LUCAT1 expression is an independent prognostic factor}

We used the univariate Cox analysis to select the related variables for multivariate Cox analysis. Following multivariate Cox analysis, high LUCAT1 expression was an independent prognostic factor for overall survival (hazard ratio: 2.35, confidence interval (CI): 1.56-3.54, $\mathrm{p}<0.001)$ and relapse-free survival (hazard ratio: 2.04, CI: 1.32-3.16, $\mathrm{p}=0.001$ ) in liver cancer (Tables III and IV).
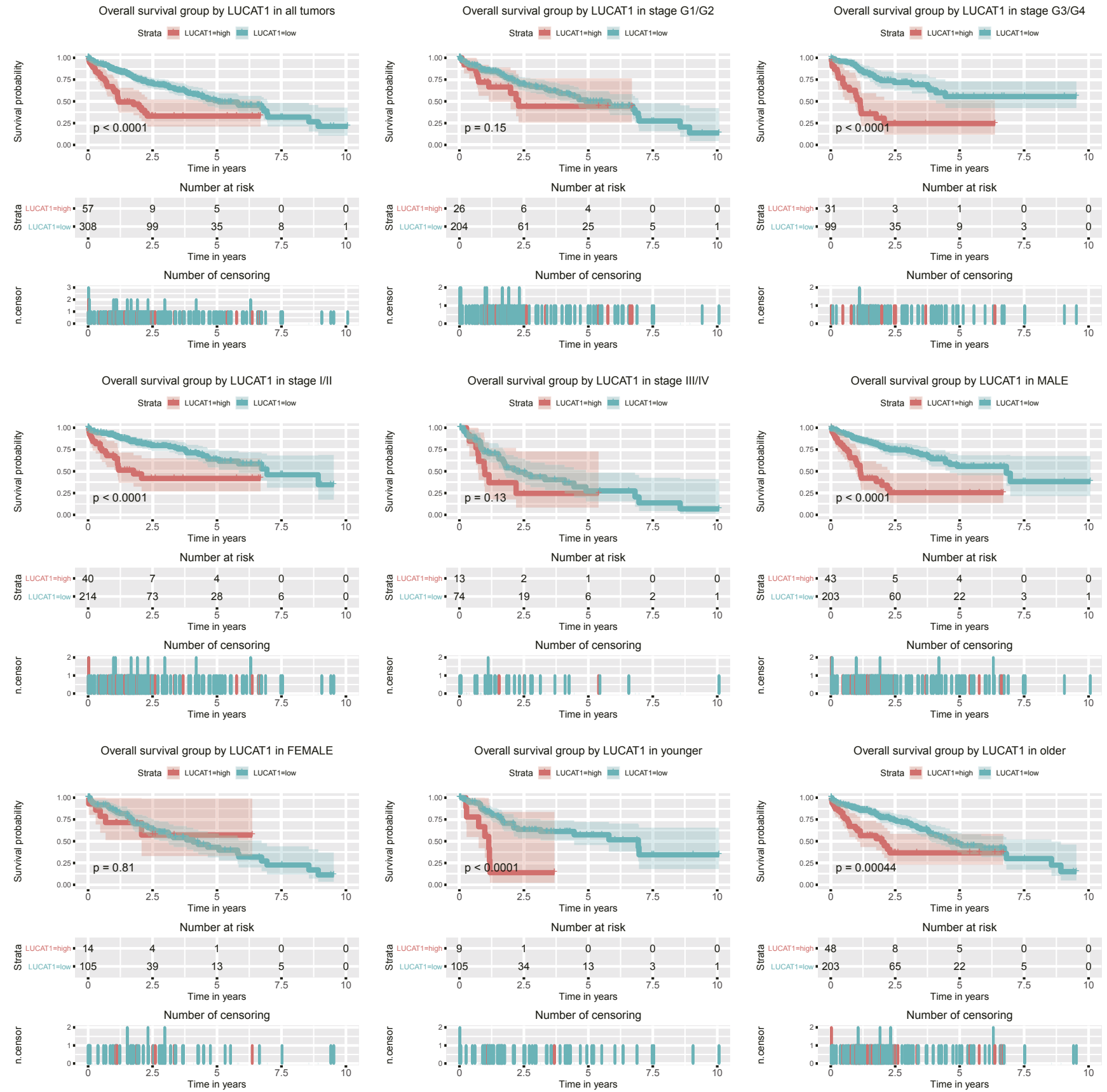

Fig. 2. High LUCAT1 expression is correlated with a shorter overall survival (OS) in patients with liver cancer, and the OS of the G1/G2, G3/ G4, stage I/II, stage III/IV, male, female, younger, and older subgroups. 

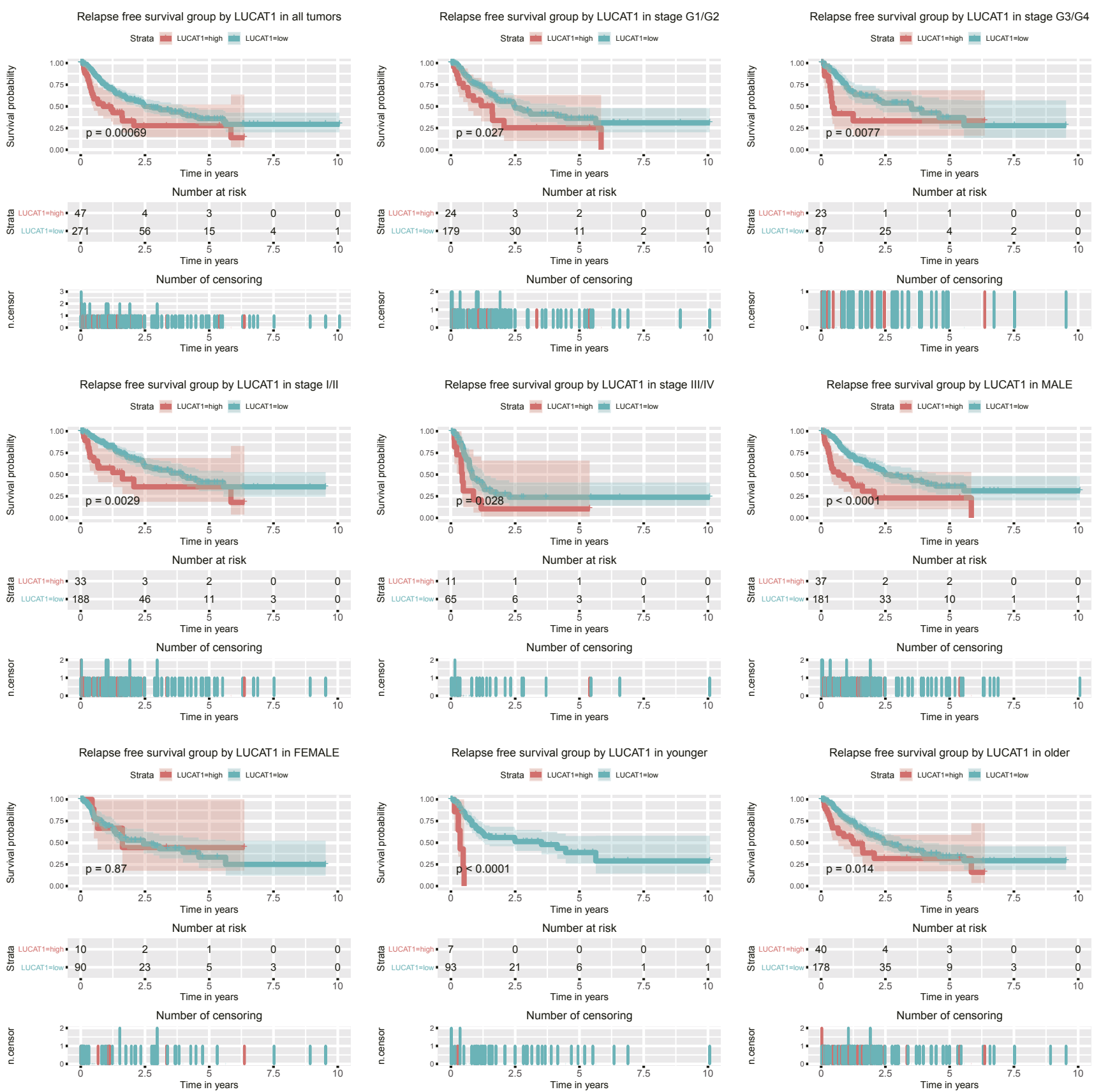

Fig. 3. High LUCAT1 expression is correlated with a shorter relapse-free survival (RFS) in patients with liver cancer, and the RFS of the G1/ G2, G3/G4, stage I/II, stage III/IV, male, female, younger, and older subgroups.

\section{Diagnostic value of LUCAT}

We performed receiver operating characteristic (ROC) analysis to determine the diagnostic value of LUCAT1 expression (Supplementary Fig. 1). LUCAT1 expression had potential diagnostic value overall $(\mathrm{AUC}=0.749)$ and was also able to distinguish noncancerous tissue from stage I cancer (AUC $=0.725)$, stage II cancer (AUC $=0.799)$, stage III cancer $(\mathrm{AUC}=0.745)$, and stage IV cancer $(\mathrm{AUC}=0.784)$.

LUCAT1-related signaling pathways and ceRNA network

To identify the LUCAT1-related signaling pathways activated in liver cancer, we conducted GSEA between low and high LUCAT1 expression data sets. Significant differences $(\mathrm{FDR}<0.25$, NOM $\mathrm{p}$-value $<0.05)$ were found in the enrichment of the MSigDB collection (h.all.v6.2.symbols. gmt), and the details are shown in Supplementary Fig. 2 and Supplementary Table I. Myc targets, reactive oxygen species pathway, DNA repair, mTORC1 signaling, and G2M checkpoint are differentially enriched in the LUCAT1 highexpression phenotype.

To explore the underlying mechanism of LUCAT1, we selected 17 downregulated DEMs and 465 upregulated DEGs between the low and high LUCAT1 expression groups. Next, we merged the DEMs, DEGs, and predicted miRNA-mRNA targets and constructed a LUCAT1-related ceRNA network (Supplementary Fig. 3). 
Table II. The correlation between LUCAT1 expression and clinical parameters

\begin{tabular}{|c|c|c|c|c|c|c|}
\hline \multirow{2}{*}{$\begin{array}{l}\text { Clinical } \\
\text { characteristics }\end{array}$} & \multirow{2}{*}{ Variable } & \multirow{2}{*}{$\begin{array}{c}\text { No. of } \\
\text { patients }\end{array}$} & \multicolumn{2}{|c|}{ LUCAT1 expression } & \multirow{2}{*}{$\chi^{2}$} & \multirow{2}{*}{$\mathrm{p}$ value } \\
\hline & & & High (\%) & Low \% & & \\
\hline \multirow[t]{2}{*}{ Age } & $<55$ & 117 & $9(15.52)$ & $108(34.62)$ & 7.3905 & 0.007 \\
\hline & $\geq 55$ & 253 & $49(84.48)$ & $204(65.38)$ & & \\
\hline \multirow[t]{2}{*}{ Gender } & Female & 121 & $14(24.14)$ & $107(34.19)$ & 1.8137 & 0.178 \\
\hline & Male & 250 & $44(75.86)$ & $206(65.81)$ & & \\
\hline \multirow[t]{3}{*}{ Histologic type } & Fibrolamellar & 3 & $0(0)$ & $3(0.96)$ & 0.5722 & 1.000 \\
\hline & Hepatocellular & 361 & $57(98.28)$ & $304(97.12)$ & & \\
\hline & Hepatocholangiocarcinoma & 7 & $1(1.72)$ & $6(1.92)$ & & \\
\hline \multirow[t]{4}{*}{ Histologic grade } & G1 & 55 & $4(6.9)$ & $51(16.56)$ & 11.5817 & 0.009 \\
\hline & G2 & 177 & $22(37.93)$ & $155(50.32)$ & & \\
\hline & G3 & 122 & $30(51.72)$ & $92(29.87)$ & & \\
\hline & G4 & 12 & $2(3.45)$ & $10(3.25)$ & & \\
\hline \multirow[t]{4}{*}{ Stage } & I & 171 & $20(37.04)$ & $151(51.54)$ & 8.0049 & 0.063 \\
\hline & II & 86 & $21(38.89)$ & $65(22.18)$ & & \\
\hline & III & 85 & $13(24.07)$ & $72(24.57)$ & & \\
\hline & IV & 5 & $0(0)$ & $5(1.71)$ & & \\
\hline \multirow[t]{5}{*}{ T classification } & $\mathrm{T} 1$ & 181 & $21(36.21)$ & $160(51.45)$ & 10.7821 & 0.022 \\
\hline & $\mathrm{T} 2$ & 94 & $23(39.66)$ & $71(22.83)$ & & \\
\hline & $\mathrm{T} 3$ & 80 & $10(17.24)$ & $70(22.51)$ & & \\
\hline & $\mathrm{T} 4$ & 13 & $4(6.9)$ & $9(2.89)$ & & \\
\hline & $\mathrm{Tx}$ & 1 & $0(0)$ & $1(0.32)$ & & \\
\hline \multirow[t]{3}{*}{$\mathrm{N}$ classification } & No & 252 & $40(68.97)$ & $212(67.95)$ & 0.7521 & 1.000 \\
\hline & N1 & 4 & $0(0)$ & $4(1.28)$ & & \\
\hline & $\mathrm{Nx}$ & 114 & $18(31.03)$ & $96(30.77)$ & & \\
\hline \multirow[t]{3}{*}{ M classification } & M0 & 266 & $42(72.41)$ & $224(71.57)$ & 0.7494 & 1.000 \\
\hline & M1 & 4 & $0(0)$ & $4(1.28)$ & & \\
\hline & $\mathrm{Mx}$ & 101 & $16(27.59)$ & $85(27.16)$ & & \\
\hline \multirow[t]{2}{*}{ Radiation therapy } & No & 338 & $54(98.18)$ & $284(97.59)$ & 0 & 1.000 \\
\hline & Yes & 8 & $1(1.82)$ & $7(2.41)$ & & \\
\hline \multirow[t]{4}{*}{ Residual tumor } & R0 & 324 & $49(87.5)$ & $275(89.29)$ & 3.4377 & 0.334 \\
\hline & $\mathrm{R} 1$ & 17 & $5(8.93)$ & $12(3.9)$ & & \\
\hline & $\mathrm{R} 2$ & 1 & $0(0)$ & $1(0.32)$ & & \\
\hline & $\mathrm{Rx}$ & 22 & $2(3.57)$ & $20(6.49)$ & & \\
\hline \multirow[t]{2}{*}{ Vital status } & Deceased & 130 & $31(53.45)$ & $99(31.63)$ & 9.298 & 0.002 \\
\hline & Living & 241 & $27(46.55)$ & $214(68.37)$ & & \\
\hline
\end{tabular}

For abbreviations see Table I.

\section{DISCUSSION}

Liver cancer is one of the most frequent malignancies with a poor prognosis. Most liver cancer patients are diagnosed at an advanced stage with no effective treatment options. Therefore, exploring novel biomarkers and understanding the underlying mechanisms may lead to promising improvements in the outcomes of liver cancer.

Recently, increasing research has revealed the critical roles of lncRNAs in physiological processes and their dysregulation in human diseases, especially cancer. Accumulating evidence illustrates that aberrant lncRNA expression is involved in various cancers. The use of lncRNAs, such as ATB, PVT1,
MALAT1, HOTAIR, and UCA1, as novel prognostic biomarkers has gained more attention.

Our team has focused on novel biomarkers in many types of cancer [18-30]. In this study, we focused on LUCAT1, a novel lncRNA, and found that LUCAT1 was significantly upregulated in liver cancer. Additionally, LUCAT1 expression correlated with age, histologic grade, $\mathrm{T}$ classification, and survival status. Moreover, patients whose cancerous tissues showed high LUCAT1 expression had shorter overall survival (OS) and relapse-free survival (RFS). Subgroup analysis revealed the specific groups in which LUCAT1 had the most prognostic value. Importantly, the Cox multivariate model showed that LUCAT1 expression plays a vital role in OS/ 
Table III. Univariate and multivariate cox analysis of overall survival.

\begin{tabular}{|c|c|c|c|c|c|c|}
\hline \multirow[b]{2}{*}{ Parameters } & \multicolumn{3}{|c|}{ Univariate analysis } & \multicolumn{3}{|c|}{ Multivariate analysis } \\
\hline & Hazard Ratio & $\begin{array}{c}95 \% \text { CI } \\
\text { (lower upper) }\end{array}$ & p-value & Hazard Ratio & $\begin{array}{c}95 \% \text { CI } \\
\text { (lower-upper) }\end{array}$ & $\mathrm{p}$-value \\
\hline Age & 1.02 & $0.7-1.48$ & 0.926 & & & \\
\hline Gender & 0.82 & $0.57-1.16$ & 0.263 & & & \\
\hline Histologic type & 0.98 & $0.27-3.63$ & 0.982 & & & \\
\hline Histologic grade & 1.05 & $0.85-1.31$ & 0.651 & & & \\
\hline Stage & 1.38 & $1.15-1.65$ & 0.001 & 0.88 & $0.7-1.1$ & 0.265 \\
\hline $\mathrm{T}$ classification & 1.65 & $1.38-1.98$ & $<0.001$ & 1.78 & $1.4-2.25$ & $<0.001$ \\
\hline $\mathrm{N}$ classification & 0.71 & $0.5-1.03$ & 0.071 & & & \\
\hline M classification & 0.70 & $0.48-1.02$ & 0.061 & & & \\
\hline Radiation therapy & 0.52 & $0.26-1.03$ & 0.061 & & & \\
\hline Residual tumor & 1.42 & $1.12-1.79$ & 0.004 & 1.44 & $1.12-1.85$ & 0.004 \\
\hline LUCAT1 & 2.58 & $1.71-3.88$ & $<0.001$ & 2.35 & $1.56-3.54$ & $<0.001$ \\
\hline
\end{tabular}

Table IV. Univariable and multivariable cox analysis of relapse free survival

\begin{tabular}{|c|c|c|c|c|c|c|}
\hline \multirow[b]{2}{*}{ Parameters } & \multicolumn{3}{|c|}{ Univariate analysis } & \multicolumn{3}{|c|}{ Multivariate analysis } \\
\hline & Hazard Ratio & $\begin{array}{c}95 \% \text { CI } \\
\text { (lower upper) }\end{array}$ & p-value & Hazard Ratio & $\begin{array}{c}\text { 95\% CI } \\
\text { (lower-upper) }\end{array}$ & $\mathrm{p}$-value \\
\hline Age & 0.89 & $0.63-1.27$ & 0.521 & & & \\
\hline Gender & 0.98 & $0.69-1.4$ & 0.919 & & & \\
\hline Histologic type & 2.03 & $0.66-6.29$ & 0.218 & & & \\
\hline Histologic grade & 0.98 & $0.8-1.21$ & 0.873 & & & \\
\hline Stage & 1.66 & $1.38-1.99$ & $<0.001$ & 1.14 & $0.88-1.47$ & 0.334 \\
\hline T classification & 1.78 & $1.49-2.12$ & $<0.001$ & 1.64 & $1.25-2.14$ & $<0.001$ \\
\hline $\mathrm{N}$ classification & 0.98 & $0.68-1.42$ & 0.926 & & & \\
\hline M classification & 1.19 & $0.8-1.78$ & 0.394 & & & \\
\hline Radiation therapy & 0.75 & $0.26-2.17$ & 0.592 & & & \\
\hline Residual tumor & 1.27 & $1.01-1.61$ & 0.042 & 1.36 & $1.07-1.73$ & 0.012 \\
\hline LUCAT1 & 2.09 & $1.35-3.22$ & 0.001 & 2.04 & $1.32-3.16$ & 0.001 \\
\hline
\end{tabular}

RFS. Moreover, ROC curves showed the diagnostic value of LUCAT1. Therefore, LUCAT1 may be a potential clinical biomarker for liver cancer.

The IncRNA LUCAT1 has been reported to be overexpressed in lung cancer [2], ovarian cancer [31], clear cell renal cell carcinoma [3], colorectal cancer [32], HPV-negative head and neck squamous cell carcinoma [7], osteosarcoma [6], esophageal squamous cell carcinoma [33], and glioma [5]. Consistent with these findings in cancer research, we found LUCAT1 overexpression in liver cancer.

Many studies have reported that LUCAT1 has an effect on the proliferation [2], migration and invasion of tumor cells [5], being involved in cell cycle [34] of many cancer cells. LUCAT1 contributes to the malignant biological behavior of cancer. Accordingly, our study found that LUCAT1 expression was associated with histologic grade, $\mathrm{T}$ classification, and vital status in liver cancer.

Importantly, LUCAT1 promotes cell proliferation by regulating p21 and p57 expression and predicts unfavorable survival in non-small cell lung cancer [2]. In addition, the Akt/ GSK-3 $\beta$ signaling pathway is also involved in the proliferation and invasion regulated by LUCAT1 [34]. Moreover, the function of LUCAT1 involves DNA methylation. LUCAT1 activates DNMT1, a DNA methylation protein, to repress the expression of tumor suppressor genes, resulting in the development of cancer [33,35]. Our study found that LUCAT1 overexpression was correlated with shorter OS and RFS in liver cancer. GSEA revealed that the potential mechanism might be involved in myc targets, reactive oxygen species pathways, DNA repair, mTORC1 signaling, and the G2M checkpoint.

The ceRNA theory has recently attracted much attention in the field of regulatory mechanisms of lncRNAs. The interplay between IncRNAs and miRNAs plays a crucial role in cancer biology. However, few studies have reported the LUCAT1related ceRNA regulatory mechanism. For example, Han et al. [6] found that LUCAT1 sponges microRNA-200 and unregulated $\mathrm{ABCB} 1$ to modulate methotrexate resistance in osteosarcoma [6]. Yu et al. found that LUCAT1 acted as a ceRNA of HOXA13 to promote the malignancy of ovarian cancer by sponging microRNA-612 [31]. Additionally, miR495-3p and miR-375 have been found in the regulatory mechanism of LUCAT1 $[5,36]$. In this study, we constructed 
the LUCAT1-related ceRNA network, parts of which have been indicated by some published research while other parts still need to be verified in the future.

To the best of our knowledge, this is the first study to identify a correlation between lncRNA LUCAT1 expression and clinical features in liver cancer based on data mining. Of note, LUCAT1 may serve as an independent prognostic factor for poor OS/RFS in liver cancer.

However, we did not utilize the LUCAT1 expression and clinical data to construct a predictive model because it is not enough to use some clinical parameters and a single gene to predict patients' prognosis. The related molecular mechanisms and multiomics of LUCAT1 still need to be elucidated in future studies.

\section{CONCLUSION}

Our study revealed the critical role of LUCAT1 overexpression in liver cancer, indicating that LUCAT1 expression is an independent predictor of poor prognosis and could be a useful biomarker for liver cancer patients.

Conflicts of interest: The authors declare that no competing interests exist.

Authors' contributions: Y.J. and Y.L. analyzed the data, designed the study and drafted the manuscript. H.C. Y.L and B.J. interpreted the results and reviewed the manuscript. All authors critically revised the manuscript and approved the final version.

Supplementary material: To access the supplementary material visit the online version of the J Gastrointestin Liver Dis at http://dx.doi. org/10.15403/jgld-356

\section{REFERENCES}

1. Gramantieri L, Baglioni M, Fornari F, et al. LncRNAs as novel players in hepatocellular carcinoma recurrence. Oncotarget 2018;9:35085-35099. doi:10.18632/oncotarget.26202

2. Sun Y, Jin SD, Zhu Q, et al. Long non-coding RNA LUCAT1 is associated with poor prognosis in human non-small lung cancer and regulates cell proliferation via epigenetically repressing p 21 and p57 expression. Oncotarget 2017;8:28297-28311. doi:10.18632/oncotarget.16044

3. Xiao H, Bao L, Xiao W, et al. Long non-coding RNA Lucatl is a poor prognostic factor and demonstrates malignant biological behavior in clear cell renal cell carcinoma. Oncotarget 2017;8:113622-113634. doi:10.18632/oncotarget.21185

4. Cao YP, Zhou J, Li WJ, et al. Long Non-Coding RNA Expression Profiles for the Characterization of Different Bladder Cancer Grade. Cell Physiol Biochem 2018;50:1154-1163. doi:10.1159/000494542

5. Gao YS, Liu XZ, Zhang YG, Liu XJ, Li LZ. Knockdown of Long Noncoding RNA LUCAT1 Inhibits Cell Viability and Invasion by Regulating miR-375 in Glioma. Oncol Res 2018;26:307-313. doi:10.37 27/096504017X15088061795756

6. Han Z, Shi L. Long non-coding RNA LUCAT1 modulates methotrexate resistance in osteosarcoma via miR-200c/ABCB1 axis. Biochem Biophys Res Commun 2018;495:947-953. doi:10.1016/j.bbrc.2017.11.121
7. Haque SU, Niu L, Kuhnell D, et al. Differential expression and prognostic value of long non-coding RNA in HPV-negative head and neck squamous cell carcinoma. Head Neck 2018;40:1555-1564. doi:10.1002/ hed.25136

8. Mootha VK, Lindgren CM, Eriksson KF, et al. PGC-1alpha-responsive genes involved in oxidative phosphorylation are coordinately downregulated in human diabetes. Nat Genet 2003;34:267-273. doi:10.1038/ng1180

9. Subramanian A, Tamayo P, Mootha VK, et al. Gene set enrichment analysis: a knowledge-based approach for interpreting genome-wide expression profiles. Proc Natl Acad Sci U S A 2005;102:15545-15550. doi:10.1073/pnas.0506580102

10. Ritchie ME, Phipson B2, Wu D, et al. limma powers differential expression analyses for RNA-sequencing and microarray studies. Nucleic Acids Res 2015;43:e47. doi:10.1093/nar/gkv007

11. Yang JH, Li JH, Shao P, Zhou H, Chen YQ, Qu LH. starBase: a database for exploring microRNA-mRNA interaction maps from Argonaute CLIP-Seq and Degradome-Seq data. Nucleic Acids Res 2011;39(Database issue):D202-D209. doi:10.1093/nar/gkq1056

12. Samur MK. RTCGAToolbox: a new tool for exporting TCGA Firehose data. PLoS One 2014;9:e106397. doi:10.1371/journal.pone.0106397

13. R Development Core Team. R: A language and environment for statistical computing. R Foundation for Statistical Computing, Vienna, Austria. 2011. Available at: https://www.R-project.org/

14. Rubio VG. Book review: ggplot2 - Elegant Graphics for Data Analysis (2nd Edition). J Stat Softw 2017;77:(Book review 2). doi:10.18637/jss. v077.b02

15. Robin X, Turck N, Hainard A, et al. pROC: an open-source package for $\mathrm{R}$ and $\mathrm{S}+$ to analyze and compare ROC curves. BMC Bioinformatics 2011;12:77. doi:10.1186/1471-2105-12-77

16. Therneau TM. A Package for Survival Analysis in S. version 2.38. 2015. Available at: https://CRAN.R-project.org/package=survival

17. Book review. Modeling Survival Data: Extending the Cox Model. Therneau TM,. Grambsch PM. Springer-Verlag, New York, 2000. Statistics in Medicine 2001;20:2053-2054. doi:10.1002/sim.956

18. Jiao Y, Fu Z, Li Y, Meng L, Liu Y. High EIF2B5 mRNA expression and its prognostic significance in liver cancer: a study based on the TCGA and GEO database. Cancer Manag Res 2018;10:6003-6014. doi:10.2147/ CMAR.S185459

19. Jiao Y, Fu Z, Li Y, Zhang W, Liu Y. Aberrant FAM64A mRNA expression is an independent predictor of poor survival in pancreatic cancer. PLoS One 2019;14:e0211291. doi:10.1371/journal.pone.0211291

20. Jiao Y, Li Y, Jiang P, Han W, Liu Y. PGM5: a novel diagnostic and prognostic biomarker for liver cancer. PeerJ 2019;7:e7070. doi:10.7717/ peerj.7070

21. Jiao Y, Li Y, Liu S, Chen Q, Liu Y. ITGA3 serves as a diagnostic and prognostic biomarker for pancreatic cancer. Onco Targets Ther 2019;12:4141-4152. doi:10.2147/OTT.S201675

22. Jiao Y, Li Y, Lu Z, Liu Y. High Trophinin-Associated Protein Expression Is an Independent Predictor of Poor Survival in Liver Cancer. Dig Dis Sci 2019;64:137-143. doi:10.1007/s10620-018-5315-X

23. Li Y, Jiao Y, Fu Z, Luo Z, Su J, Li Y. High miR-454-3p expression predicts poor prognosis in hepatocellular carcinoma. Cancer Manag Res 2019;11:2795-2802. doi:10.2147/CMAR.S196655

24. Hou L, Zhang X, Jiao Y, et al. ATP binding cassette subfamily B member 9 (ABCB9) is a prognostic indicator of overall survival in ovarian cancer. Medicine (Baltimore) 2019;98:e15698. doi:10.1097/ MD.0000000000015698 
25. Jiao Y, Li Y, Fu Z, et al. OGDHL Expression as a Prognostic Biomarker for Liver Cancer Patients. Disease Markers 2019;2019: 9037131. doi:10.1155/2019/9037131

26. Li Y, Jiao Y, Li Y, Liu Y. Expression of La Ribonucleoprotein Domain Family Member 4B (LARP4B) in Liver Cancer and Their Clinical and Prognostic Significance. Disease Markers 2019;2019: 1569049. doi:10.1155/2019/1569049

27. Li Y, Jiao Y, Luo Z, Li Y, Liu Y. High peroxidasin-like expression is a potential and independent prognostic biomarker in breast cancer. Medicine (Baltimore) 2019;98:e17703. doi:10.1097/ MD.0000000000017703

28. Zhang X, Cui Y, He M, Jiao Y, Yang Z. Lipocalin-1 Expression as a Prognosticator Marker of Survival in Breast Cancer Patients. Breast Care 2019. doi:10.1159/000503168

29. Cui Y, Jiao Y, Wang K, He M, Yang Z. A new prognostic factor of breast cancer: High carboxyl ester lipase expression related to poor survival. Cancer Genet 2019;239:54-61. doi:10.1016/j.cancergen.2019.09.005

30. Cai H, Jiao Y, Li Y, Yang Z, He M, Liu Y. Low CYP24A1 mRNA expression and its role in prognosis of breast cancer. Sci Rep 2019;9:13714. doi:10.1038/s41598-019-50214-Z

31. Yu H, Xu Y, Zhang D, Liu G. Long noncoding RNA LUCAT1 promotes malignancy of ovarian cancer through regulation of miR-612/HOXA13 pathway. Biochem Biophys Res Commun 2018;503:2095-2100. doi:10.1016/j.bbrc.2018.07.165

32. Chen $\mathrm{Y}, \mathrm{Yu} \mathrm{X}, \mathrm{Xu} \mathrm{Y}$, Shen $\mathrm{H}$. Identification of dysregulated lncRNAs profiling and metastasis-associated lncRNAs in colorectal cancer by genome-wide analysis. Cancer Med 2017;6:2321-2330. doi:10.1002/ cam4.1168

33. Yoon JH, You BH, Park CH, Kim YJ, Nam JW, Lee SK. The long non-coding RNA LUCAT1 promotes tumorigenesis by controlling ubiquitination and stability of DNA methyltransferase 1 in esophageal squamous cell carcinoma. Cancer Lett 2018;417:47-57. doi:10.1016/j.canlet.2017.12.016

34. Zheng Z, Zhao F, Zhu D, et al. Long Non-Coding RNA LUCAT1 Promotes Proliferation and Invasion in Clear Cell Renal Cell Carcinoma Through AKT/GSK-3beta Signaling Pathway. Cell Physiol Biochem 2018;48:891-904. doi:10.1159/000491957

35. Ling XX, Zhang HQ, Liu JX, et al. LncRNA LUCAT1 Activation Mediated by the Down-regulation of DNMT1 Is Involved in Cell Apoptosis Induced by PM2.5. Biomed Environ Sci 2018;31:608-612. doi:10.3967/bes2018.082

36. Wang LN, Zhu XQ, Song XS, Xu Y. Long non-coding RNA lung cancer associated transcript 1 promotes proliferation and invasion of clear cell renal cell carcinoma cells by negatively regulating miR-495-3p. J Cell Biochem 2018;119:7599-7609. doi:10.1002/jcb.27099 
Supplementary Table I. Gene sets enriched in phenotype high

\begin{tabular}{lrrr}
\hline Gene set name & NES & NOM p-val & FDR q-val \\
\hline HALLMARK_MYC_TARGETS_V2 & -1.9075 & 0.02549 & 0.06957 \\
HALLMARK_MYC_TARGETS_V1 & -1.8553 & 0.02519 & 0.04965 \\
HALLMARK_REACTIVE_OXIGEN_SPECIES_PATHWAY & -1.7538 & 0.0332 & 0.06838 \\
HALLMARK_DNA_REPAIR & -1.6681 & 0.03953 & 0.09399 \\
HALLMARK_MTORC1_SIGNALING & -1.6427 & 0.04921 & 0.09195 \\
HALLMARK_G2M_CHECKPOINT & -1.6052 & 0.04717 & 0.0981 \\
\hline
\end{tabular}

NES: normalized enrichment score; NOM: nominal; FDR: false discovery rate. Gene sets with NOM

p-val $<0.05$ and FDR q-val $<0.25$ are considered as significant. 

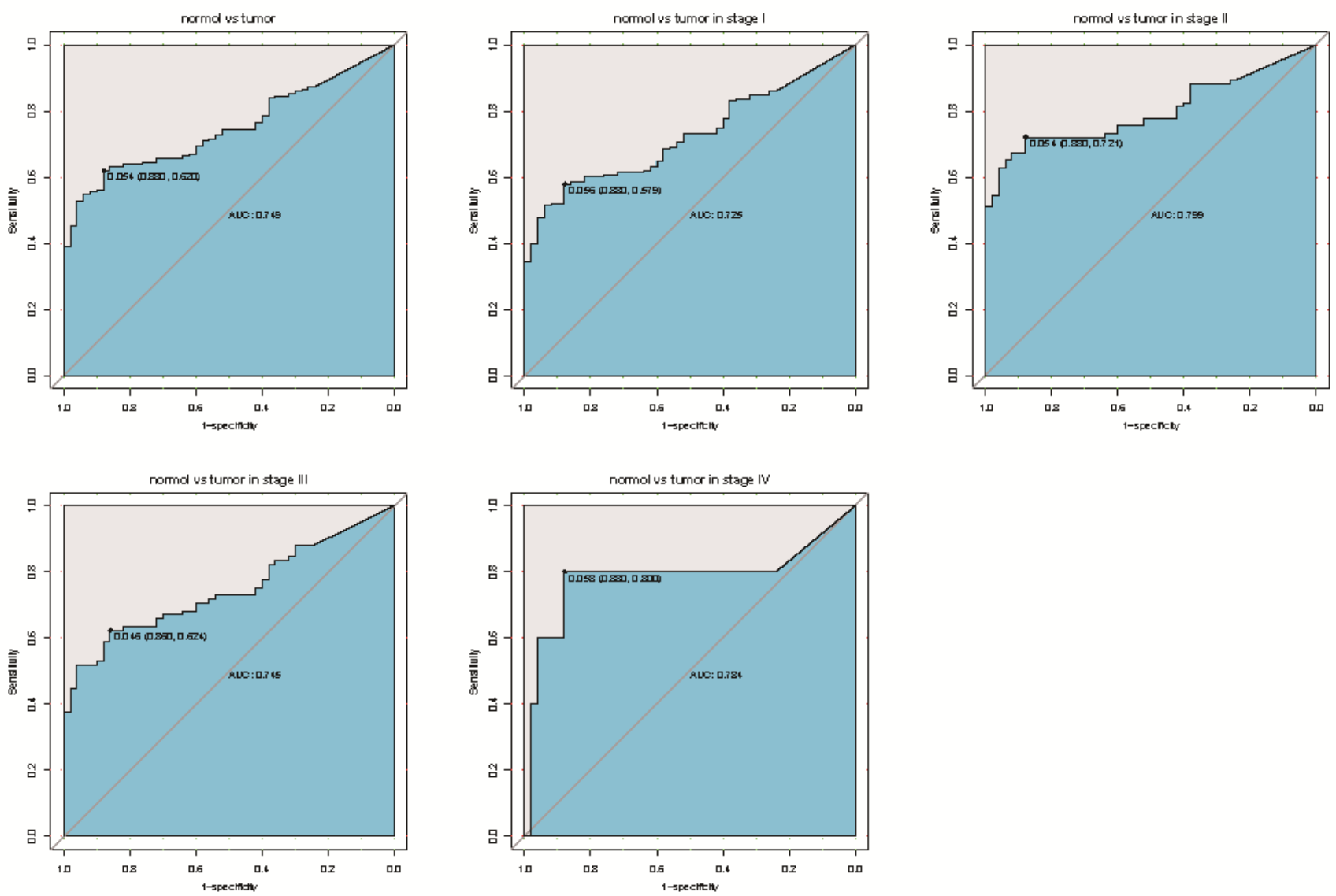

Supplementary Fig. 1. ROC analysis of the performance of LUCAT1 expression in the identification of cancerous vs. normal tissues in all patients and subgroup analysis of patients with different stages of liver cancer. 

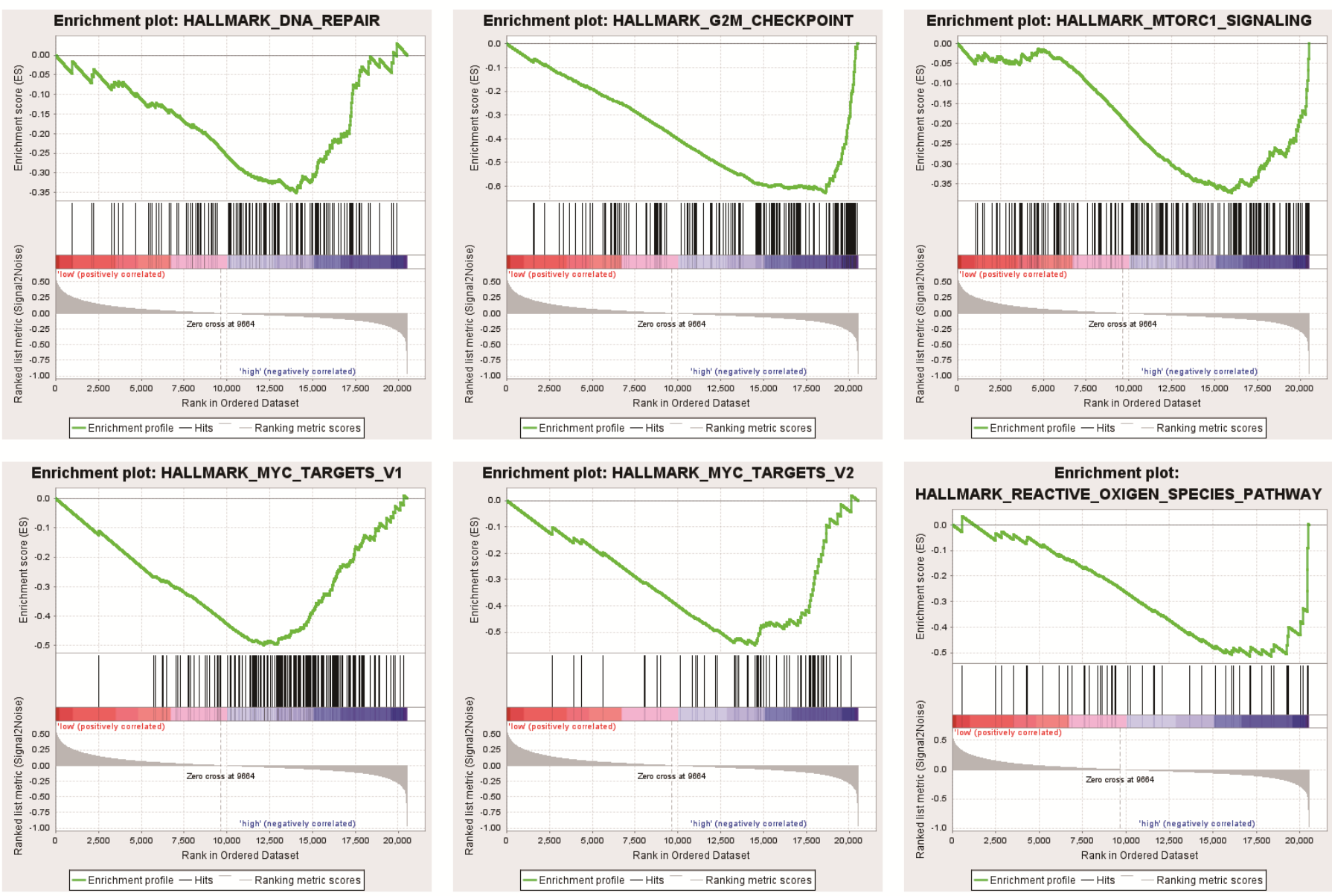

Supplementary Fig. 2. Enrichment plots from GSEA. Myc targets, the reactive oxygen species pathway, DNA repair, mTORC1 signaling, and the G2M checkpoint are differentially enriched in LUCAT1-related liver cancer. 


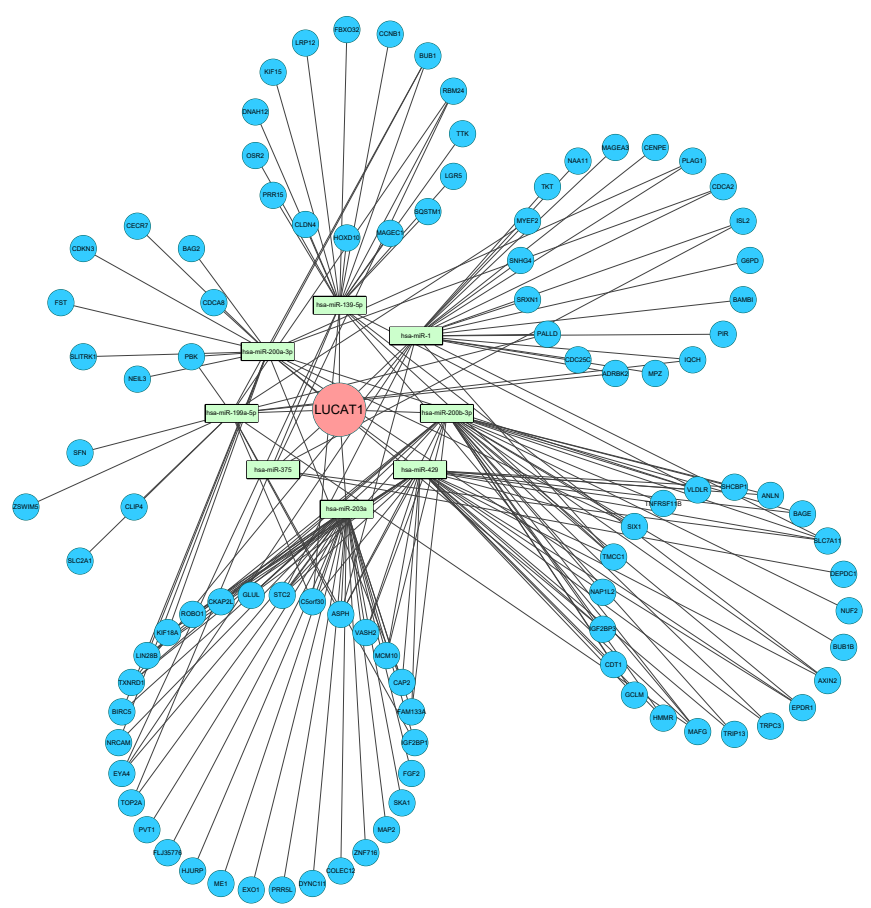

Supplementary Fig. 3. LUCAT1-related ceRNA network. The red circle represents the lncRNA LUCAT1, the green rectangles represent the microRNAs, and the blue circles represent the mRNAs 
